\title{
BARRIER PROPERTIES OF LIPID BILAYERS COMPOSED OF LECITHINS WITH ODD CHAIN FATTY ACIDS
}

\author{
S. SALVATI and G. SER LUPI-CRESCENZI \\ Istituto Superiore di Sanita, Viale Regina Elena 299, 00161 Roma (Italia) \\ J. DE GIER \\ Laboratory of Biochemistry, State University of Utrecht, Padualaan 8 , \\ Utrecht (The Netherlands)
}

Received October 27th, 1978 accepted December 5th, 1978

Lecithins with fatty acid chain length of 17 carbon atoms and different degrees of unsaturation were synthesized. The thermotropic behaviour and barrier function of derived liposomal bilayers were studied.

\section{Introduction}

Recent experiments showed that the normally low concentration of odd chain fatty acids in brain can be changed by dietary means. Increased amounts of these unusual acids were found in newborn and weaning rats whose mothers had been fed an odd chain lipid fraction extracted from yeast grown on $n$-alkanes [1]. Furthermore studies on the behavioural development of these young rats showed a lowering of the age of appearance of a number of reflexes linked to myelinisation when compared to animals fed with a control diet [2]. The correlation between these findings yielded the question whether the barrier function of the membrane bilayer may be changed by the presence of odd paraffin chains. In this context this paper describes results of a comparative study on the barrier properties of liposome systems prepared from lecithins with odd and even paraffin chains.

\section{Materials and methods}

Odd chain fatty acids - cis-9-heptadecanoic and cis,cis-9,12-heptadecadienoic acid - were isolated as described before [3] . 1,2-Diheptadecenoyl-sn-3-phosphocholine ( $C_{17: 1} / C_{17: 1}$-phosphatidylcholine) and 1,2-diheptadecadienoyl-sn-3-phosphocholine $\left(C_{17: 2} / C_{17: 2}\right.$-phosphatidylcholine) were synthesized according to 
Warner et al. [4] . 1,2-Diheptadecanoyl-sn-3-phosphocholine $\left(\mathrm{C}_{17: 0} / \mathrm{C}_{17: 0}\right.$-phos-

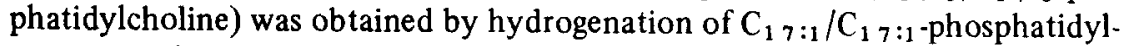
choline. Reference components with even numbered chains were prepared by similar routes.

The calorimetric analyses were performed on a Perkin Elmer DSC 2 B instrument as described by van Dijck et al. [5].

Freeze fracturing electron microscopy was done according to Ververgaert et al. $[6]$.

Comparative permeability measurements concerning non-electroly te permeability of liposomal bilayers were carried out under conditions outlined in earlier studies [7].

\section{Results and discussion}

In view of the well-known anomalous melting temperatures of odd chain free fatty acids when compared to the even numbered components (compare Fig. 2), our interest was directed to the thermotropic behaviour of the bilayer systems of the odd chain lecithins. The calorimetric scan of liposomes prepared of pure 17:0/ 17:0-phosphatidylcholine in Fig. 1 shows, in agreement with results on systems of other saturated lecithins, a main transition and at lower temperature a much smaller pretransition. The main transition at $47.8 \pm 0.4^{\circ} \mathrm{C}$ represents most likely the change from the gel to the liquid crystalline condition. The pretransition is probably related to the change from a flat $L_{\beta} 1$ phase to a rippled $P_{\beta} 1$ phase [8]. Fig. 3 shows a freeze fracture electronmicrograph of the liposomes quenched at about $45^{\circ} \mathrm{C}$, which demonstrates the characteristic wave pattern of the $\mathrm{P}_{\beta} 1$ phase.

In Fig. 2 a comparison is made between the temperature of the gel to liquid crystalline transition of liposomes composed of pure 17:0/17:0-phosphatidylcholine and these transition temperatures found for the systems of even numbered lecithins. It can be concluded that in contrast to the melting points of the free fatty acids the melting temperature of the odd chains in the bilayers is in line with those of the even numbered systems. In agreement with this finding the analyses of the $\mathrm{C}_{17: 1} / \mathrm{C}_{17: 1}$-phosphatidylcholine system showed a gel to liquid crystalline transition at $-27.6 \pm 0.5^{\circ} \mathrm{C}$ which is intermediate between the transitions of dipalmitoleoyl-and dioleoyl phosphatidylcholine liposomes at $-36^{\circ} \mathrm{C}$ and $-14^{\circ} \mathrm{C}$ respectively [7]. Furthermore the enthalpy change involved in the melting of the chains in the 17:0/17:0-phosphatidylcholine system was calculated to be $9.2 \mathrm{kcal} /$ mol in comparison with $8.6 \mathrm{kcal} / \mathrm{mol}$ for dipalmitoyl- and 10.7 for distearoylphosphatidylcholine liposomes.

Figure 1 shows in addition to the calorimetric scan of pure 17:0/17:0-phosphatidylcholine the scans of equimolar mixtures of this component with dipalmitoyl-and dimyristoyl-phosphatidylcholine. A broadening of the phase transition peaks can be noticed but there is no phase separation. This confirms earlier observa- 

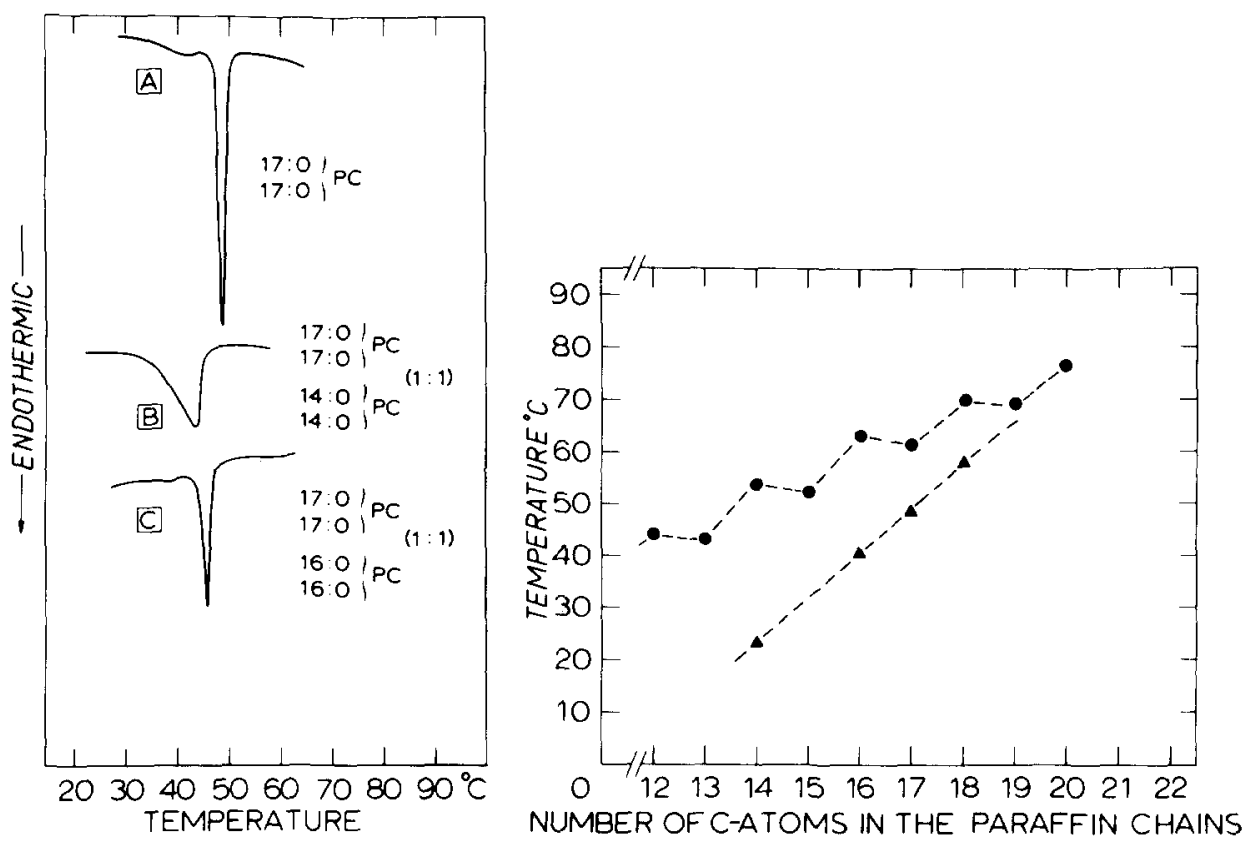

Fig. 1. Thermotrophic behaviour of liposome systems of pure 17:0/17:0-phosphatidylcholine and of equimolar mixtures of this lipid with 14:0/14:0-phosphatidylcholine and 16:0/16:0phosphatidylcholine, registered by differential scanning calorimetry.

Fig. 2. Solid-liquid transition temperatures of odd and even numbered carbon chains in different systems: ( - . . - $\bullet$ ) melting temperatures of free fatty acids; data taken from H.A. Sober (Ed.), 'Handbook of Biochemistry', The Chemical Rubber Co. Cleveland, OH, 2nd Ed., 1970; (4- . . . - $)$ gel-liquid crystalline transition of liposome systems prepared of pure lecithins.

tions that monotectic behaviour is only obtained when the difference in the paraffin chain length is at least 4 carbon atoms $[6,9]$. Studies on mixtures of diheptadecanoyl phosphatidylcholine and cholesterol showed that as in other cholesterol-lecithin mixtures [10] the cholesterol withdraws lecithin molecules from the phase transition and that above $40 \mathrm{~mol} \%$ of cholesterol the phase transition is eliminated completely.

The actual barrier function of the liposomal bilayers in the liquid crystalline condition was tested by comparative measurements on the permeability for glycerol and erythritol. Liposomes prepared in $150 \mathrm{mM} \mathrm{KCl}$ were dispersed in isotonic solutions of the two non-electrolytes and the initial swelling rate was registered as a function of temperature. The result shown in Fig. 4 indicates that there is a gradual decrease in permeability both for glycerol and erythritol when the paraffin chain length increases. The data found for the system with the odd chains are found exactly in between the values for the systems with adjoining even chains. Compara- 


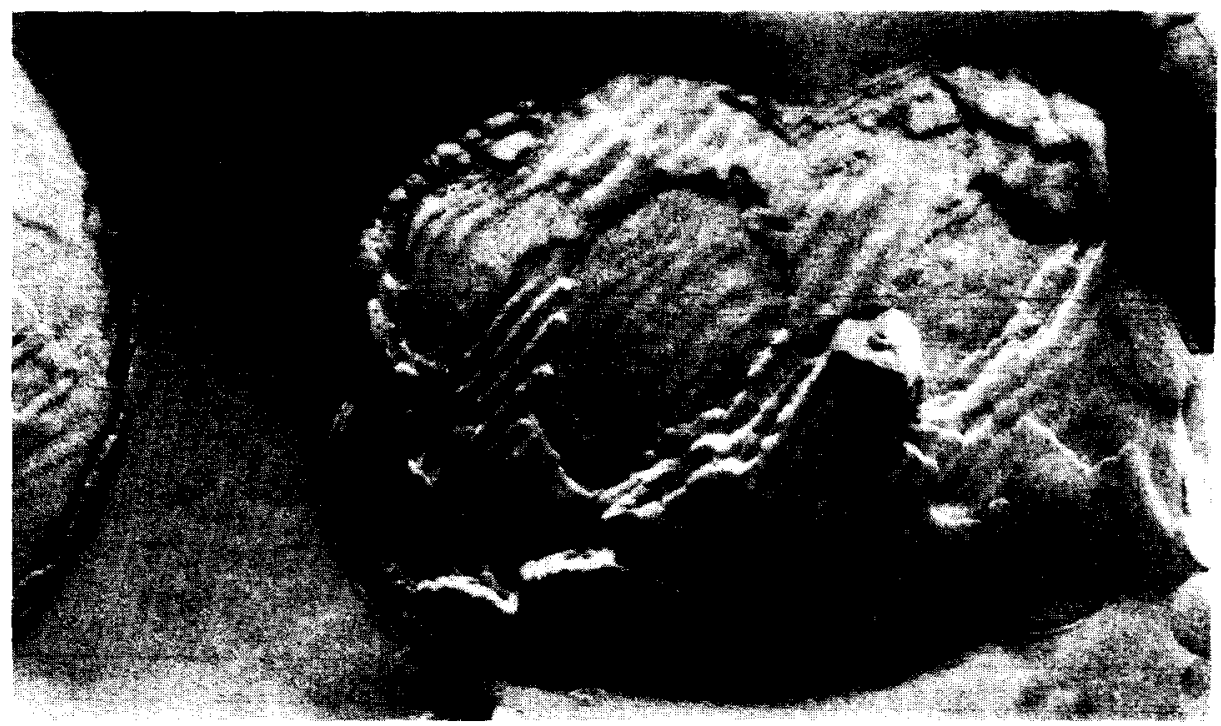

Fig. 3. Freeze fracture electron micrograph showing the rippled structure of the $P_{\beta} 1$ phase of 17:0/17:0 phosphatidylcholine liposomes.

tive studies on the liposomes of 17:0/17:0-phosphatidylcholine, $C_{17: 1} / C_{17: 1}$-phosphatidylcholine and $C_{17: 2} / C_{17: 2}$-phosphatidylcholine showed, in direct resemblance with systems with even numbered chains [7], an increase in permeability with

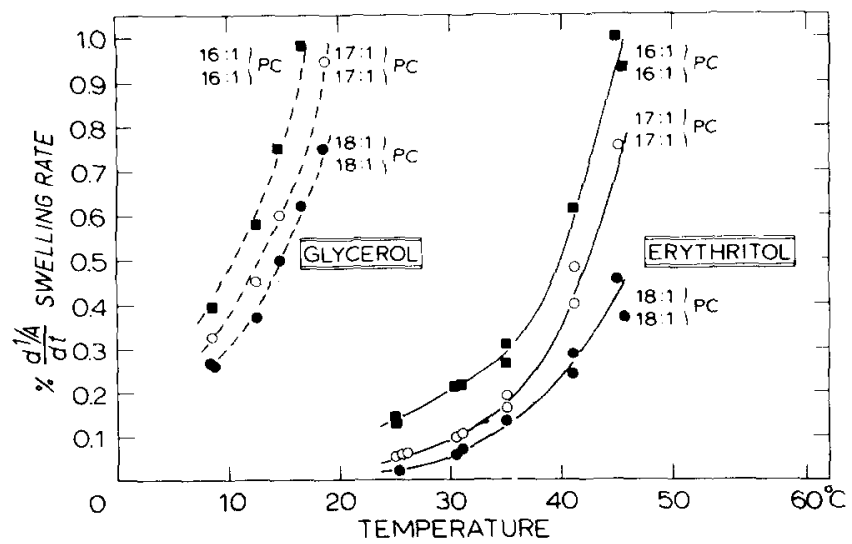

Fig. 4. Initial swelling rate of liposomes composed of 16:1/16:1 phosphatidylcholine, 17:1/ $17: 1$ phosphatidylcholine and 18:1/18:1 phosphatidylcholines as a function of temperature. To enhance the osmotic value of the structures all the systems contained 4 mol\% of phosphatidic acid. The swelling rates were measured in isotonic glycerol (-....) and in isotonic ery thritol. 
increasing unsaturation, whereas the permeability was largely reduced upon introduction of cholesterol into the lipid bilayer.

Summarizing it can be concluded that the thermotropic and barrier properties of bilayers with odd paraffin chains are easily predicted by interpolation between the properties of systems composed of lipids with adjacent even numbered chains. In the present study no anomalous effects could be detected. In view of this result the dietary effects discussed in the Introduction are not easily explained in terms of changes in the lipid bilayer functions of the myelin membrane as a consequence of the incorporation of odd chain fatty acid chains. Therefore future studies should be directed to the possibility of specific lipid-protein interactions of membrane proteins with the odd chain. Furthermore it can be speculated that the brain function is affected by specific prostaglandins sythesized from the poly-unsaturated odd chain fatty acids in the diet $[11,12]$.

\section{Acknowledgements}

The authors gratefully acknowledge Dr. R.A. Demel and Dr. P.W.M. van Dijck for valuable advice, Miss J.G. Mandersloot and Miss M. Tieman for their practical assistance and Dr. A.J. Verkleij for carrying out the freeze fracturing experiment.

\section{References}

1 M.P. Bernardini, S. Salvati, G. Serlupi-Crescenzi, B. Tagliamonte and G. Tomassi, Nutr. Rep. Int., 17 (1978) 137.

2 S. Gozzo, A. Oliverio, S. Salvati, G. Serlupi-Crescenzi, B. Tagliamonte and G. Tomassi, Nutr. Rep. Int., 17 (1978) 357.

3 R. Dommarc, E. Rea, S. Salvati and G. Serlupi-Crescenzi, Riv. Soc. Alim. (1978) in press.

4 T.G. Warner and A.A.Benson, J. Lipid Res., 18 (1977) 548.

5 P.W.M. van Dijck, B. de Kruijff, L.L.M. van Deenen, J. de Gier, and R.A. Demel, Biochim. Biophys. Acta, 455 (1976) 576.

6 P.H.J.Th. Ververgaert, A.J. Verkleij, P.F. Elbers and L.L.M. van Deenen, Biochim. Biophys. Acta, 311 (1973) 320.

7 J. de Gier, J.G. Mandersloot and L.L.M. van Deenen, Biochim. Biophys. Acta 150 (1968) 666.

8 M.J. Janiak, D.M. Small and G.G. Shipley, Biochemistry, 15 (1976) 4575.

9 M.C. Philips, B.D. Ladbrooke and D. Cbapman, Biochim. Biophys. Acta, 196 (1960) 35.

10 R.A. Demel and B. de Kruijff, Biochim. Biophys. Acta, 457 (1976) 109.

11 D.A. van Dorp, Ann. N.Y. Acad. Sci., 180 (1971) 181.

12 H. Sihlenk, I'ed. Proc., 31 (1972) 1430. 